\title{
Distorted copulas: constructions and tail dependence
}

\author{
Fabrizio Durante, Rachele Foschi and Peter Sarkoci \\ Department of Knowledge-Based Mathematical Systems \\ Johannes Kepler University, A-4040 Linz, Austria \\ e-mail: fabrizio.durante@jku.at \\ Dipartimento di Matematica \\ Università degli Studi di Roma "La Sapienza", I-00185 Rome, Italy \\ e-mail: foschi@mat.uniroma1.it \\ Department of Knowledge-Based Mathematical Systems \\ Johannes Kepler University, A-4040 Linz, Austria \\ e-mail: peter.sarkoci@jku.at, peter.sarkoci@gmail.com
}

Received: December 17, 2008. Accepted: May 7, 2009

\begin{abstract}
Given a copula $C$, we examine under which conditions on an order isomorphism $\psi$ of $[0,1]$, the distortion $C_{\psi}:[0,1]^{2} \rightarrow[0,1], C_{\psi}(x, y)=$ $\psi\left(C\left(\psi^{-1}(x)\right), \psi^{-1}(y)\right)$, is again a copula. In particular, when the copula $C$ is totally positive of order 2 , we give a sufficient condition on $\psi$ which ensures that any distortion of $C$ by means of $\psi$ is again a copula. The presented results allow us to introduce in a more flexible way families of copulas exhibiting different behaviour in the tails.

Keywords: copula, distorted probability, isomorphic transformation, tail dependence, $\mathrm{TP}_{2}$ property.

AMS Subject Classification: 60E05, 62H20.
\end{abstract}

${ }^{*}$ Corresponding author. Tel. +43 7322468 9191, Fax +43 73224681351. 


\section{Introduction}

A (bivariate) copula is a distribution function on $\mathbb{I}^{2}=[0,1]^{2}$ whose univariate margins are uniformly distributed. Copulas have received a great popularity due to the celebrated Sklar's Theorem, stating that every bivariate distribution function can be represented by means of some suitable copula and its marginal distribution functions. For an introduction to copula theory and some of its applications, we refer to Schweizer and Sklar (2006); Joe (1997); Nelsen (2006); McNeil et al. (2005); Salvadori et al. (2007).

The growing importance of copulas in statistical models has originated several methods for generating new classes of such functions. The final goal of these investigations is to obtain more flexible families of multivariate distribution functions, having a variety of interesting properties like tail dependencies, asymmetries, wide range of association.

In this paper, we concentrate our attention on a transformation acting on bivariate copulas. Given a copula $C$ and an increasing bijection $\psi: \mathbb{I} \rightarrow \mathbb{I}$, we consider the function $C_{\psi}: \mathbb{I}^{2} \rightarrow \mathbb{I}$ defined by

$$
C_{\psi}(x, y)=\psi\left(C\left(\psi^{-1}(x), \psi^{-1}(y)\right)\right) .
$$

Such a transformation has been considered several times in the literature, under different names like distortion or transformation of a copula by means of $\psi$. It has originated from the study of distorted probability distribution functions (especially, power distortions), and has been considered by several authors like Frees and Valdez (1998); Durrleman et al. (2000); Genest and Rivest (2001); Durante and Sempi (2005a); Klement et al. (2005b,a); Morillas (2005); Charpentier (2008); Alvoni et al. (2009). In reliability theory, Bassan and Spizzichino (2005b,a) studied this kind of transformation in order to introduce the so-called bivariate ageing function that is used for the definition of bivariate notion of ageing (see, also, Durante and Spizzichino (2009); Nappo and Spizzichino (2009)). Crane and van der Hoek (2008) used distortions of copulas to produce a heavy tailed portfolio loss distribution in the context of synthetic Collateralized Debt Obligations (CDOs).

The study of distortions is of general interest since they can be used for generating, in a flexible way, new families of copulas. In order to do this, we need conditions on $\psi$ warranting that, for a given $C, C_{\psi}$ is still a copula. Moreover, it would be also of interest to investigate how some dependence properties of $C$ change or are preserved under these distortions.

The paper is organized as follows. In section 2, we revisit the distortion of copulas from a new perspective, which includes the results obtained in the literature. In sections 3 and 4, we mainly focus on some statistical aspects 
of copulas. In particular, in section 3 , the $\mathrm{TP}_{2}$ property is studied, both as a dependence property and for its implications on the preservation of the 2 -increasing property under distortion. In section 4 , dependence on the tails of the copula is investigated. More precisely, we analyze how the tail dependence properties are modified under distortions. These results will show the usefulness of distortion for generating flexible statistical models. The presented results may be seen in the frame of the more general case of distorted probabilities. Section 5 is devoted to some comments clarifying this possible extension.

\section{Revisiting distortions of copulas}

A large part of literature about distortions mainly focuses on the determination of the class of all increasing bijections $\psi$ such that the mapping given by eq. (1.1) transforms any copula $C$ into another copula. Here we are rather interested in the (generally, larger) class of all increasing bijections $\psi$ such that, for a given copula $C, C_{\psi}$ is still a copula.

Before revisiting the notion of distortion, we introduce some notations that will be useful in the rest of the paper.

Definition 2.1. A mapping $C: \mathbb{I}^{2} \rightarrow \mathbb{I}$ is a copula if it satisfies the following properties:

(C1) $C$ is increasing in each variable,

(C2) $C(x, 1)=C(1, x)=x$ for every $x \in \mathbb{I}$,

(C3) $C$ is 2-increasing, that is, for every $x_{1}, y_{1}, x_{2}, y_{2} \in \mathbb{I}$, it satisfies

$$
\begin{aligned}
V[C]\left(\left[x_{1}, x_{2}\right]\right. & \left.\times\left[y_{1}, y_{2}\right]\right) \\
& =C\left(x_{1}, y_{1}\right)+C\left(x_{2}, y_{2}\right)-C\left(x_{1}, y_{2}\right)-C\left(x_{2}, y_{1}\right) \geq 0 .
\end{aligned}
$$

Important examples of copulas are $M(x, y)=\min (x, y), \Pi(x, y)=x y$ and $W(x, y)=\max (x+y-1,0)$ describing, respectively, comonotone dependence, independence and countermonotone dependence between two random variables. The symbol $\mathcal{C}$ stands for the class of all copulas.

Within this paper, whenever we write rectangle, we mean a subset of the unit square of type $R=\left[x_{1}, x_{2}\right] \times\left[y_{1}, y_{2}\right]$. For a binary operation $C: \mathbb{I}^{2} \rightarrow \mathbb{I}$ we define the $C$-volume of the rectangle $R$ by

$$
V[C](R)=C\left(x_{1}, y_{1}\right)-C\left(x_{2}, y_{1}\right)-C\left(x_{1}, y_{2}\right)+C\left(x_{2}, y_{2}\right) .
$$


Thus, any $C: \mathbb{I}^{2} \rightarrow \mathbb{I}$ is 2-increasing if each rectangle has a nonnegative $C$-volume.

We call an order isomorphism any increasing bijective transformation of $\mathbb{I}$. The set of all order isomorphisms is denoted by $\mathcal{I}$. If $\psi$ is an order isomorphism and $C$ a mapping from $\mathbb{I}^{2}$ to $\mathbb{I}$, then we call $\psi$-transform of $C$, or $\psi$-distortion of $C$, the mapping $C_{\psi}: \mathbb{I}^{2} \rightarrow \mathbb{I}$ defined by (1.1).

It is obvious that all the $\psi$-distortions of a copula $C$ satisfy $(\mathrm{C} 1)$ and (C2), but not necessarily the 2 -increasing property. Thus they are (continuous) semi-copulas, but not necessarily copulas (see Bassan and Spizzichino (2001, 2005b); Durante and Sempi (2005a,b)). From another point of view, any of such distortions can be considered as the semi-copula associated with a suitable distorted probability (as clarified by Durante and Spizzichino (2009)).

Given $C \in \mathcal{C}$, let $\mathcal{I}(C)$ be the set of all order isomorphisms which, being applied to $C$, give rise to another copula. Precisely

$$
\mathcal{I}(C)=\left\{\psi \in \mathcal{I} \mid C_{\psi} \in \mathcal{C}\right\} .
$$

By the literature cited above, it is well known that, regardless of $C$, the set $\mathcal{I}(C)$ contains $\mathcal{I}_{c x}$, the set of all convex bijections on $\mathbb{I}$. However, as a key observation stimulating this investigation, for a fixed copula $C$, the inclusion of $\mathcal{I}_{c x}$ into $\mathcal{I}(C)$ may be strict. For example, it is well known that $\mathcal{I}(M)=\mathcal{I}$, while $\mathcal{I}(W)=\mathcal{I}_{c x}$ (see, for example, Morillas (2005)).

Example 2.2. If $C$ is an Archimedean copula with an additive generator $f: \mathbb{I} \rightarrow[0,+\infty[$, i.e.

$$
C(x, y)=f^{(-1)}(f(x)+f(y)),
$$

then $\mathcal{I}(C)$ consists of all $\psi \in \mathcal{I}$ such that $f \circ \psi^{-1}$ is convex.

Example 2.3. If $C$ is an extreme value copula (see, e.g., Nelsen (2006)), then $\mathcal{I}(C)$ also contains all the power functions $\psi(t)=t^{\alpha}$ for every $\alpha>0$.

These facts spur us to investigate whether, fixed a copula $C$, it is possible to construct the set $\mathcal{I}(C)$ or, at least, to find its elements that are not convex. As we will see at the end of the section, these results will be useful for constructing families of copulas starting with some fixed $C$.

For this end, we need some preliminary considerations.

Lemma 2.4. (Marshall and Olkin, 1979, Proposition 4.B.2) Let $A$ be an interval of $\mathbb{R}$ and let $f: A \rightarrow \mathbb{R}$. If $f$ is convex and increasing, then, for every $a_{1}, a_{2}, a_{3}, a_{4} \in A$ such that

$$
a_{1} \leq \min \left(a_{2}, a_{3}\right) \leq \max \left(a_{2}, a_{3}\right) \leq a_{4},
$$


and $a_{1}+a_{4} \geq a_{2}+a_{3}$, we have

$$
f\left(a_{1}\right)+f\left(a_{4}\right) \geq f\left(a_{2}\right)+f\left(a_{3}\right) .
$$

Then, we have to consider the following definition on $\mathcal{I}$, which can be obtained from Hardy et al. (1952).

Definition 2.5. Let $\varphi, \psi$ be in $\mathcal{I}$. We say that $\varphi$ is less convex than $\psi$ (and we write $\varphi \leq_{\mathrm{cx}} \psi$ ) if $\psi \circ \varphi^{-1}$ is convex.

It can be shown quite easily, that the relation $\leq_{\mathrm{cx}}$ is an ordering on $\mathcal{I}$, i.e. it is reflexive, transitive and antisymmetric. Moreover, we have that $\varphi \leq_{\mathrm{cx}} \operatorname{Id}_{\mathbb{I}}$ if, and only if, $\varphi$ is concave, and, analogously, $\operatorname{Id}_{\mathbb{I}} \leq_{\mathrm{cx}} \varphi$ if, and only if, $\varphi$ is convex, where $\operatorname{Id}_{\mathbb{I}}$ is the identity function on $\mathbb{I}$. More results about the convex ordering among probability distribution functions can be derived from Chan et al. (1990), where different assumptions are given on the functions $\varphi$ and $\psi$ (see also (Mehmet and Yalçın, 2007)), and from Müller and Stoyan (2002) and Shaked and Shanthikumar (2007), where this order is considered under different names (e.g., likelihood ratio order).

Here we would like just to stress that, by using the previous definition, we have that $\varphi \leq_{\mathrm{cx}} \psi$ if, and only if, $\varphi \circ \psi^{-1}$ is concave, i.e., for all $c, d \in \mathbb{I}$ where $c<d$ and for every $y \in] c, d]$

$$
\frac{\varphi \circ \psi^{-1}(y)-\varphi \circ \psi^{-1}(c)}{y-c} \geq \frac{\varphi \circ \psi^{-1}(d)-\varphi \circ \psi^{-1}(c)}{d-c},
$$

which is equivalent to the fact that

$$
\frac{\varphi(x)-\varphi(a)}{\varphi(b)-\varphi(a)} \geq \frac{\psi(x)-\psi(a)}{\psi(b)-\psi(a)}
$$

holds for all $a, b \in \mathbb{I}$ where $a<b$ and for every $x \in] a, b]$.

The following theorem is the main result of this section. It states a closure property of the sets $\mathcal{I}(C)$ with respect to the afore-mentioned convex ordering. It will allow us to single out further (non convex) isomorphisms belonging to $\mathcal{I}(C)$, once we have found one.

Theorem 2.6. Let $\varphi, \psi \in \mathcal{I}$ such that $\varphi \leq_{\mathrm{cx}} \psi$. If $\varphi$ is an element in $\mathcal{I}(C)$, then so is $\psi$.

Proof. First note that, in order to prove that $\psi \in \mathcal{I}(C)$, it is enough to verify the 2 -increasingness of $C_{\psi}$, as conditions (C1) and (C2) are trivially preserved. 
If $R=\left[x_{1}, x_{2}\right] \times\left[y_{1}, y_{2}\right]$ is a rectangle of $\mathbb{I}^{2}$ and $\psi$ is an order isomorphism, we denote $R^{\psi^{-1}}=\left[\psi^{-1}\left(x_{1}\right), \psi^{-1}\left(x_{2}\right)\right] \times\left[\psi^{-1}\left(y_{1}\right), \psi^{-1}\left(y_{2}\right)\right]$. Now, let $C$ be a copula. We denote by $C_{i, j}^{R}=C\left(x_{i}, y_{j}\right)$, where $i, j \in\{1,2\}$, the value that $C$ assumes on the vertex $\left(x_{i}, y_{j}\right)$ of the rectangle $R$. We introduce the set

$$
\operatorname{Rq}(C)=\left\{\left(C_{1,1}^{R}, C_{1,2}^{R}, C_{2,1}^{R}, C_{2,2}^{R}\right) \in \mathbb{I}^{4} \mid R \text { is a rectangle },\right\}
$$

that consists of all quadruples of values of $C$ in the vertices of all possible rectangles of $\mathbb{I}^{2}$. For every $\psi \in \mathcal{I}$, it is not difficult to prove that

$$
\operatorname{Rq}(C)=\left\{\left(C_{1,1}^{R^{\psi^{-1}}}, C_{1,2}^{R^{\psi^{-1}}}, C_{2,1}^{R^{\psi^{-1}}}, C_{2,2}^{R^{\psi^{-1}}}\right) \in \mathbb{I}^{4} \mid R \text { is a rectangle }\right\},
$$

where, for $i, j \in\{1,2\}, C_{i, j}^{R^{\psi^{-1}}}=C\left(\psi^{-1}\left(x_{i}\right), \psi^{-1}\left(y_{j}\right)\right)$ denotes the value that $C$ assumes on the vertex $\left(\psi^{-1}\left(x_{i}\right), \psi^{-1}\left(y_{j}\right)\right)$ of the rectangle $R^{\psi^{-1}}$. Therefore, in order to prove that $\psi \in \mathcal{I}$ is also an element of $\mathcal{I}(C)$, we only have to prove that, for every $(a, b, c, d) \in \operatorname{Rq}(C)$,

$$
\psi(a)-\psi(b)-\psi(c)+\psi(d) \geq 0 .
$$

Now, let $(a, b, c, d)$ be in $\operatorname{Rq}(C)$ and let $\varphi \in \mathcal{I}(C)$. Thanks to the monotonicity of $\varphi$, we have

$$
\varphi(a) \leq \min (\varphi(b), \varphi(c)) \leq \max (\varphi(b), \varphi(c)) \leq \varphi(d) .
$$

Moreover, since $\varphi \in \mathcal{I}(C)$,

$$
\varphi(a)-\varphi(b)-\varphi(c)+\varphi(d) \geq 0 .
$$

From Lemma 2.4, this inequality is preserved when the following affine mapping $T_{a, d}$ is applied to each term:

$$
T_{a, d}:[\varphi(a), \varphi(d)] \rightarrow[\psi(a), \psi(d)], x \mapsto \frac{x-\varphi(a)}{\varphi(d)-\varphi(a)}(\psi(d)-\psi(a))+\psi(a) .
$$

Therefore, we obtain that

$$
\left(T_{a, d} \circ \varphi\right)(a)-\left(T_{a, d} \circ \varphi\right)(b)-\left(T_{a, d} \circ \varphi\right)(c)+\left(T_{a, d} \circ \varphi\right)(d) \geq 0 .
$$

We can check easily that $\left(T_{a, d} \circ \varphi\right)(a)=\psi(a)$ and $\left(T_{a, d} \circ \varphi\right)(d)=\psi(d)$. Moreover, by assumption $\varphi \leq_{\mathrm{cx}} \psi$ and, in particular, by (2.2), we have

$$
\left(T_{a, d} \circ \varphi\right)(b) \geq \psi(b) \quad \text { and } \quad\left(T_{a, d} \circ \varphi\right)(c) \geq \psi(c) .
$$

Therefore also

$$
\psi(a)-\psi(b)-\psi(c)+\psi(d) \geq 0,
$$

which is the desired assertion. 
To put Theorem 2.6 differently, every $\mathcal{I}(C)$ is an upper set with respect to convex ordering $\leq_{\mathrm{cx}}$.

As a consequence of Theorem 2.6, we may obtain the following result, already known in the literature.

Corollary 2.7. For every $C \in \mathcal{C}, \mathcal{I}_{c x} \subseteq \mathcal{I}(C)$.

Proof. Let $C$ be a copula. Clearly $\operatorname{Id}_{\mathbb{I}} \in \mathcal{I}(C)$. Moreover, by the definition of the relation $\leq_{\mathrm{cx}}$ and Theorem 2.6 it follows easily that $\operatorname{Id}_{\mathbb{I}} \leq_{\mathrm{cx}} \psi$ if and only if $\psi$ is convex.

The following theorem shows a further procedure to obtain members of $\mathcal{I}(C)$, starting with two other members belonging to this class. The result is based on the closure property of sets $\mathcal{I}(C)$ with respect to convex combinations.

Theorem 2.8. Let $C$ be a copula. If $\varphi, \psi$ are members of $\mathcal{I}(C)$, then so is $\alpha \varphi+(1-\alpha) \psi$ for any $\alpha \in \mathbb{I}$.

Proof. The assumption $\varphi, \psi \in \mathcal{I}(C)$ is equivalent to

$$
\begin{aligned}
& \varphi\left(C_{1,1}^{R}\right)-\varphi\left(C_{1,2}^{R}\right)-\varphi\left(C_{2,1}^{R}\right)+\varphi\left(C_{2,2}^{R}\right) \geq 0, \\
& \psi\left(C_{1,1}^{R}\right)-\psi\left(C_{1,2}^{R}\right)-\psi\left(C_{2,1}^{R}\right)+\psi\left(C_{2,2}^{R}\right) \geq 0,
\end{aligned}
$$

for every rectangle $R$. Multiplying the first inequality by $\alpha$, the second one by $(1-\alpha)$ and adding them up yields the inequality

$$
\varrho\left(C_{1,1}^{R}\right)-\varrho\left(C_{1,2}^{R}\right)-\varrho\left(C_{2,1}^{R}\right)+\varrho\left(C_{2,2}^{R}\right) \geq 0,
$$

where $\varrho=\alpha \varphi+(1-\alpha) \psi$. Since this inequality also holds for any rectangle $R$, we have $\varrho \in \mathcal{I}(C)$.

Summarizing, given a copula $C$ and $\psi \in \mathcal{I}(C)$, Theorems 2.6 and 2.8 might suggest two methods for constructing other elements in $\mathcal{I}(C)$ :

- take all $\varphi \in \mathcal{I}$ such that $\psi \leq_{\text {cx }} \varphi$,

- take all convex combinations between $\psi$ and such a $\varphi$ or any convex $\varphi \in \mathcal{I}$.

Both these methods may be applied for constructing families of copulas, based on $C$, by means of suitable $\psi$-transforms as above. In particular, they are "relevant" (i.e. do not produce just convex isomorphisms) when $\psi$ or $\varphi$ is not convex. In the next, we will see a way to obtain a possibly not convex $\psi \in \mathcal{I}(C)$. 


\section{The $\mathbf{T P}_{2}$ property under distortions}

This section aims at analysing how copulas satisfying a special dependence property called $\mathrm{TP}_{2}$ can be transformed into other $\mathrm{TP}_{2}$ copulas by means of suitable distortions. This fact is relevant from a statistical point of view: it does mean that, starting with a given element in $\mathcal{C}$, we can construct a family of copulas sharing a strong dependence property. Furthermore, from a more formal point of view, the preservation of the $\mathrm{TP}_{2}$ property guarantees the preservation of the 2-increasing property.

First of all, we recall that, given two intervals $A$ and $B$ in $\mathbb{R}$, a function $K: A \times B \rightarrow \mathbb{R}$ is said to be totally positive of order 2 (shortly, $\mathrm{TP}_{2}$ ) if, for any $x_{1}, x_{2}, y_{1}, y_{2} \in \mathbb{R}$ such that $x_{1} \leq x_{2}, y_{1} \leq y_{2}$,

$$
K\left(x_{1}, y_{1}\right) K\left(x_{2}, y_{2}\right) \geq K\left(x_{1}, y_{2}\right) K\left(x_{2}, y_{1}\right) .
$$

Analogously, $K: A \times B \rightarrow \mathbb{R}$ is said to be reverse regular of order 2 (shortly, $\mathrm{RR}_{2}$ ) if (3.1) holds with the reverse inequality sign. For more details, see Karlin and Rinott (1980a,b).

If $K$ is a probability distribution or a survival function, then the $\mathrm{TP}_{2}$ (respectively, $\mathrm{RR}_{2}$ ) property of $K$ corresponds to a strong notion of positive (respectively, negative) dependence between the random variables, usually called corner set monotonicity (compare with Harris (1970)). Moreover, it is known that this dependence property can be expressed in terms of the $\mathrm{TP}_{2}$ (respectively, $\mathrm{RR}_{2}$ ) property of the copula associated with the distribution function (see e.g. Nelsen (2006)).

Here, starting with a copula $C$ satisfying the $\mathrm{TP}_{2}$ property, we would like to find conditions on the transformation $\psi$ such that $C_{\psi}$ is also $\mathrm{TP}_{2}$. Preliminarily, we state the following result.

Lemma 3.1. Let $C: \mathbb{I}^{2} \rightarrow \mathbb{I}$ be increasing in each place. If $C$ is $T P_{2}$, then it is 2-increasing.

Proof. Suppose that $C$ is $T P_{2}$. Then, for every $x_{1}, x_{2}, y_{1}, y_{2}$ in $[0,1], x_{1} \leq x_{2}$ and $y_{1} \leq y_{2}$, we have that

$$
C\left(x_{1}, y_{1}\right) C\left(x_{2}, y_{2}\right) \geq C\left(x_{1}, y_{2}\right) C\left(x_{2}, y_{1}\right) .
$$

Because the logarithm function is strictly increasing, it follows that

$$
\log C\left(x_{1}, y_{1}\right)+\log C\left(x_{2}, y_{2}\right) \geq \log C\left(x_{1}, y_{2}\right)+\log C\left(x_{2}, y_{1}\right),
$$

i.e. $\log C$ is 2-increasing. Now, we recall that if $H$ is a 2-increasing and monotonic function and $\phi$ is convex and increasing, then $\phi \circ H$ is monotonic and 
2-increasing (see (Marshall and Olkin, 1979, page 151)). In particular, by applying this result to the exponential function and to the 2-increasing and monotonic function $\log \circ C$, we obtain that $C=\exp (\log C)$ is 2-increasing, which is the desired assertion.

Theorem 3.2. Let $C$ be a $T P_{2}$ copula. Let $\psi \in \mathcal{I}$. If $\left.\psi \circ \operatorname{exp:~]~}-\infty, 0\right] \rightarrow$ $[0,1]$ is log-convex, then

(i) $C_{\psi}$ is $T P_{2}$;

(ii) $\psi \in \mathcal{I}(C)$.

Proof. In order to prove (i), given a rectangle $R=\left[x_{1}, x_{2}\right] \times\left[y_{1}, y_{2}\right]$ of $\mathbb{I}^{2}$ we set

$$
a_{i j}=C\left(\psi^{-1}\left(x_{i}\right), \psi^{-1}\left(y_{j}\right)\right) .
$$

By definition every $a_{i j} \in \mathbb{I}$ and $a_{11} \leq \min \left(a_{12}, a_{21}\right) \leq \max \left(a_{12}, a_{21}\right) \leq a_{22}$. Since $C$ is $\mathrm{TP}_{2}$, it follows that $a_{11} a_{22} \geq a_{12} a_{21}$, which implies

$$
\log a_{11}+\log a_{22} \geq \log a_{12}+\log a_{21} .
$$

Now, by assumption $\gamma:]-\infty, 0] \rightarrow]-\infty, 0], \gamma(t)=\log \left(\psi\left(e^{t}\right)\right)$ is convex and increasing, and, by applying Lemma 2.4 to each term of inequality (3.3), we obtain

$$
\log \left(\psi\left(a_{11}\right)\right)+\log \left(\psi\left(a_{22}\right)\right) \geq \log \left(\psi\left(a_{12}\right)\right)+\log \left(\psi\left(a_{21}\right)\right),
$$

which, in its turn, implies

$$
\psi\left(a_{11}\right) \psi\left(a_{22}\right) \geq \psi\left(a_{12}\right) \psi\left(a_{21}\right) .
$$

Thus, $C_{\psi}$ is $\mathrm{TP}_{2}$. Finally, from Lemma 3.1 it follows that $C_{\psi}$ is also 2increasing and, since $C_{\psi}$ satisfies also conditions (C1) and (C2) of Definition $2.1, C_{\psi}$ is a copula.

Note that the condition $\log \circ \psi \circ \exp$ convex is sometimes referred to as geometric convexity of $\psi$ (compare with Saminger-Platz et al. (2008)).

Remark 3.3. Log-convexity also plays a key role in characterizing univariate ageing notions. For example, we say that a survival distribution function $\bar{F}$ is Decreasing Failure Rate (DFR) when it is log-convex (compare, e.g., with Lai and Xie (2006)). This means that we might construct isomorphisms $\psi$ that satisfy the assumptions of Theorem 3.2 just by taking $\psi=\bar{F} \circ(-\log )$, where $\bar{F}$ is a suitable DFR survival distribution. By using this fact, we can obtain the following examples corresponding to (versions of) Weibull, Gompertz and Lomax survival distribution functions: 
- $\psi(t)=\exp \left(-(-\log (t))^{\alpha}\right)$, where $\left.\left.\alpha \in\right] 0,1\right]$;

- $\psi(t)=\exp \left(-\frac{\alpha^{-\log (t)}-1}{\log (\alpha)}\right)$, where $\left.\left.\alpha \in\right] 0,1\right]$;

- $\psi(t)=(1-\log (t))^{-\alpha}$, where $\alpha>0$.

For a fixed $C$, the sufficient condition of Theorem 3.2 need not be necessary. In fact, the copula $M$ is $\mathrm{TP}_{2}$ and it coincides with any of its transformation $M_{\psi}$, apart from the properties of $\psi \in \mathcal{I}$. However, for the class of strict Archimedean copulas, which can be obtained as a distortion of the copula $\Pi$, we have the following characterization.

Corollary 3.4. Let $\psi \in \mathcal{I}$. Then $\Pi_{\psi}$ is a $T P_{2}$ copula if, and only if, $\psi \circ \exp$ is log-convex.

Proof. Suppose that $\Pi_{\psi}$ is a $\mathrm{TP}_{2}$ copula. Then, $\Pi_{\psi}=C$ is a strict Archimedean copula additively generated by $f(t)=-\log \left(\psi^{-1}(t)\right)$. Now, it is known from Proposition 6.1 by Bassan and Spizzichino (2005b) (compare also with Avérous and Dortet-Bernadet (2004)) that $C$ is $\mathrm{TP}_{2}$ if, and only if, $f^{-1}$ is log-convex, which is equivalent to $t \mapsto \log \left(\psi\left(e^{-t}\right)\right)$ convex on $[0,+\infty[$, that is $\psi \circ \exp$ log-convex on ] $-\infty, 0]$.

Now, a remarkable fact is derived from Theorem 3.2 by considering that every power function $\psi \in \mathcal{I}$ has the property that $\psi \circ \exp$ is log-convex.

Corollary 3.5. Let $C$ be a $T P_{2}$ copula and $\psi \in \mathcal{I}, \psi(t)=t^{\alpha}$ for $\alpha>0$. Then $C_{\psi}$ is a $T P_{2}$ copula.

This observation is of a great importance since such transformations allow us to construct several parametric families of copulas starting with a known copula $C$ that is $\mathrm{TP}_{2}$.

Example 3.6. For every $\theta \in[-1,1]$, let us consider the Farlie-GumbelMorgenstern family of copulas whose elements are given by

$$
C_{\theta}(x, y)=x y+\theta x y(1-x)(1-y) .
$$

Every $C_{\theta}$ is $T P_{2}$ when $\theta \in[0,1]$. Let us consider $\psi(t)=t^{\alpha}$ for every $\alpha>0$ and the transformed copulas $\left(C_{\theta}\right)_{\psi}$, that we denote by $C_{\alpha, \theta}$. We have that

$$
C_{\alpha, \theta}(x, y)=x y\left[1+\theta\left(1-x^{\frac{1}{\alpha}}\right)\left(1-y^{\frac{1}{\alpha}}\right)\right]^{\alpha}
$$

defines a family of copulas that are $\mathrm{TP}_{2}$ when $\theta \in[0,1]$ (compare also with Arnold et al. (2008)). 
Example 3.7. Let $C$ be a semilinear copula (compare with Durante (2006); Durante et al. (2008)), i.e. there exists $f: \mathbb{I} \rightarrow \mathbb{I}$ that is strictly increasing and continuous with $\frac{f(t)}{t}$ decreasing on $\left.] 0,1\right]$ and $f(1)=1$, such that

$$
C(x, y)=\min (x, y) f(\max (x, y)) .
$$

Let us consider $\psi(t)=t^{\alpha}$ for every $\alpha>0$. Since $C$ is $T P_{2}$, then $C_{\psi}$ is a copula for every $\alpha>0$. Actually, the distorted copula $C_{\psi}$ is again a semilinear copula generated by $g(t)=f^{\alpha}\left(t^{1 / \alpha}\right)$.

\section{Tail dependence coefficients under distortions}

A possible reason for adding new parameters to already known copulas lies in producing families that exhibit some more flexible properties. In particular, copulas with different tail behaviour are usually required for building stochastic models for estimating extreme and risky events (Joe, 1997; McNeil et al., 2005; Salvadori et al., 2007). In this section, we show how the distortions of a given copula $C$ may modify the tail behaviour of $C$, as measured by its tail dependence coefficients. Specifically, we state formulas linking the original tail dependence coefficients of $C$ and the ones obtained from some distortion of $C$.

We recall that, given two continuous random variables $X$ and $Y$ whose distribution functions are $F_{X}$ and $F_{Y}$, respectively, the upper tail dependence coefficient $\lambda_{U}$ of $(X, Y)$ is defined by

$$
\lambda_{U}=\lim _{t \rightarrow 1^{-}} \mathbb{P}\left(Y>F_{Y}^{[-1]}(t) \mid X>F_{X}^{[-1]}(t)\right)
$$

and the lower tail dependence coefficient $\lambda_{L}$ by

$$
\lambda_{L}=\lim _{t \rightarrow 0^{+}} \mathbb{P}\left(Y \leq F_{Y}^{[-1]}(t) \mid X \leq F_{X}^{[-1]}(t)\right),
$$

provided that the above limits exist in $\mathbb{I}$. These two coefficients, which are of great importance in the study of a tail behaviour of a random pair (see Joe (1997); Nelsen (2006)), can be represented in terms of the copula associated with $(X, Y)$.

Proposition 4.1. Let $X$ and $Y$ be continuous random variables with copula $C$. If $\lambda_{U}$ and $\lambda_{L}$ defined by (4.1) and (4.2) exist and take values in $\mathbb{I}$, then

$$
\begin{aligned}
& \lambda_{L}=\lim _{x \rightarrow 0^{+}} \frac{C(x, x)}{x}, \\
& \lambda_{U}=\lim _{x \rightarrow 1^{-}} \frac{1-2 x+C(x, x)}{1-x}=2-\lim _{x \rightarrow 1^{-}} \frac{1-C(x, x)}{1-x} .
\end{aligned}
$$


Moreover, if $\lambda_{U}$ and $\lambda_{L}$ exist and are finite, then

$$
\lambda_{L}=\delta_{C}^{\prime}\left(0^{+}\right) \quad \text { and } \quad \lambda_{U}=2-\delta_{C}^{\prime}\left(1^{-}\right),
$$

where $\delta_{C}: \mathbb{I} \rightarrow \mathbb{I}$ given by $\delta_{C}(x)=C(x, x)$ is the diagonal section of $C$.

Here we state our results related to the tail dependence coefficients of the distorted copula.

Proposition 4.2. Let $C$ be a copula such that $\lambda_{L}(C)$ exists and is finite. Let $\psi \in \mathcal{I}(C)$. If, for some $\alpha>0$,

$$
\left.\lim _{t \rightarrow 0^{+}} \frac{\psi(t)}{t^{\alpha}}=b \in\right] 0,+\infty[.
$$

Then $\lambda_{L}\left(C_{\psi}\right)=\left(\lambda_{L}(C)\right)^{\alpha}$.

Proof. Let $C$ be a copula with diagonal section $\delta_{C}$. If $\delta_{C}=0$ on $[0, \varepsilon]$ for a small $\varepsilon>0$, then we easily obtain that $\lambda_{L}\left(C_{\psi}\right)=0=\left(\lambda_{L}(C)\right)^{\alpha}$. Otherwise, suppose that $\delta_{C}$ is strictly increasing on $[0, \varepsilon]$ for some small $\varepsilon>0$. Taking into account (4.3), the lower tail dependence coefficient for $C_{\psi}$ can be expressed as

$$
\lambda_{L}\left(C_{\psi}\right)=\lim _{x \rightarrow 0^{+}} \frac{\psi(C(x, x))}{\psi(x)}=\lim _{x \rightarrow 0^{+}}\left(\frac{\psi(C(x, x))}{[C(x, x)]^{\alpha}} \cdot \frac{[C(x, x)]^{\alpha}}{x^{\alpha}} \cdot \frac{x^{\alpha}}{\psi(x)}\right) .
$$

Therefore, from the given assumptions, $\lambda_{L}\left(C_{\psi}\right)=\left[\lambda_{L}(C)\right]^{\alpha}$.

Proposition 4.3. Let $C$ be a copula such that $\lambda_{U}(C)$ exists and is finite. Let $\psi \in \mathcal{I}(C)$. If, for some $\alpha>0$,

$$
\left.\lim _{t \rightarrow 1^{-}} \frac{1-\psi(t)}{(1-t)^{\alpha}}=b \in\right] 0,+\infty[
$$

then $\lambda_{U}\left(C_{\psi}\right)=2-\left(2-\lambda_{U}(C)\right)^{\alpha}$.

Proof. Let $C$ be a copula with diagonal section $\delta_{C}$. Then, $\delta_{C}$ is strictly increasing on $[1-\varepsilon, 1]$ for some small $\varepsilon>0$.

By taking into account (4.5), the upper tail dependence coefficient of $C_{\psi}$ can be expressed as

$$
\lambda_{U}\left(C_{\psi}\right)=2-\lim _{x \rightarrow 1^{-}} \frac{1-\psi(C(x, x))}{1-\psi(x)}=2-\lim _{x \rightarrow 1^{-}} \frac{1-\psi\left(\delta_{C}(x)\right)}{1-\psi(x)} .
$$


If, for some $\left.\alpha>0, \lim _{t \rightarrow 1^{-}} \frac{1-\psi(t)}{(1-t)^{\alpha}}=b \in\right] 0,+\infty[$, then

$$
\lim _{x \rightarrow 1^{-}} \frac{1-\psi\left(\delta_{C}(x)\right)}{1-\psi(x)}=\lim _{x \rightarrow 1^{-}}\left(\frac{1-\psi\left(\delta_{C}(x)\right)}{\left(1-\delta_{C}(x)\right)^{\alpha}} \cdot \frac{\left(1-\delta_{C}(x)\right)^{\alpha}}{(1-x)^{\alpha}} \cdot \frac{(1-x)^{\alpha}}{1-\psi(x)}\right) .
$$

Therefore, $\lambda_{U}\left(C_{\psi}\right)=2-\left(2-\lambda_{U}(C)\right)^{\alpha}$.

Example 4.4. For every $\theta \in[-1,1]$, let us consider the Farlie-GumbelMorgenstern family $\left\{C_{\theta}\right\}$ of copulas whose elements are given by (3.5). If we consider $\widetilde{\psi} \in \mathcal{I}, \widetilde{\psi}(t)=e^{-(-\log t)^{\alpha}}$ for every $\left.\alpha \in\right] 0,1[$. It can be showed that $\widetilde{\psi}$ satisfies the assumptions of Theorem 3.2 and Proposition 4.3. Thus, $\left(C_{\theta}\right)_{\widetilde{\psi}}$ is a modification of the Farlie-Gumbel-Morgenstern copula having, additionally, upper tail dependence coefficient equal to $2-2^{\alpha}$.

For the case of strict Archimedean copulas, the above results can be stated in the following simpler forms.

Corollary 4.5. Let $C(x, y)=f^{-1}(f(x)+f(y))$ be an Archimedean copula additively generated by $f$.

(i) If $\left.t e^{\alpha f(t)} \stackrel{t \rightarrow 0^{+}}{\longrightarrow} b \in\right] 0,+\infty\left[\right.$ for some $\alpha>0$, then $\lambda_{L}(C)=0$.

(ii) If $\left.\frac{1-t}{\left(1-e^{-f(t)}\right)^{\alpha}} \stackrel{t \rightarrow 1^{-}}{\longrightarrow} b \in\right] 0,+\infty\left[\right.$ for some $\alpha>0$, then $\lambda_{U}(C)=2-2^{\alpha}$.

Proof. The proof follows easily by the Propositions 4.2 and 4.3 and by the fact that any Archimedean copula $C(x, y)=f^{-1}(f(x)+f(y))$ can be represented as a distortion $\Pi_{\psi}$ of the independence copula $\Pi$ (that has upper and lower tail dependence coefficient equal to 0$)$ with $\psi=f^{-1} \circ(-\log )$.

More general results about the tail dependence coefficients of an Archimedean copulas are given by Juri and Wüthrich $(2002,2003)$ and Charpentier and Segers $(2007,2009)$ in terms of regularly varying properties of the additive generator.

\section{Concluding remarks}

In previous sections, we have obtained several results concerning the socalled distortions of a given copula. Here, we would like to clarify how these results may be reformulated and reinterpreted in the more general context of bivariate distribution functions.

We start by a simple observation. Let us consider a bivariate continuous distribution function $F$ and an order isomorphism $\psi \in \mathcal{I}$. Suppose that 
$F_{\psi}=\psi \circ F$ is a distribution function. If $\mathbb{P}_{F}$ is the probability measure generated by $F$ on the Borel sets of $\mathbb{R}^{2}$, then $F_{\psi}$ is simply the distribution function of the probability measure $\psi \circ \mathbb{P}_{F}$. Generalized measures obtained by means of the distortion of a probability measure are usually known as distorted probabilities (Denneberg, 1994).

Now, let $C$ be the copula of $F$. It is quite easy to prove that the copula of $F_{\psi}$ is simply $C_{\psi}$ (see, e.g., Durante and Sempi (2005a)). Moreover, the following result can be also stated.

Proposition 5.1. Let us consider a bivariate continuous distribution function $F$ and an order isomorphism $\psi \in \mathcal{I}$. Let $C$ be the copula of $F$ and let $\mathbb{P}_{F}$ be the probability measure induced by $F$ on $\mathbb{R}^{2}$. The following statements are equivalent:

- $F_{\psi}(x, y)=\psi(F(x, y))$ is a distribution function,

- $\psi \circ \mathbb{P}_{F}$ is a probability measure,

- $\psi \in \mathcal{I}(C)$.

Thus, investigations about distorted copulas can be as well applied to the cases of distortions of probabilities and distribution functions.

Finally, notice that, under suitable assumptions, the distortions $F_{\psi}$ obtained from any $\psi \in \mathcal{I}(C)$ have a quite distinguished property: they describe all the bivariate distribution functions having the same level sets as $F$ (see, for example, Durante and Spizzichino (2009); Nappo and Spizzichino (2009)). Interesting statistical motivations for defining distribution functions by means of level sets have been examined by Arnold et al. (2008).

\section{Acknowledgments}

We would like to express our gratitude to an Anonymous Reviewer for her/his comments that help us in providing a better presentation of this manuscript.

Part of this work was conducted when the first two authors were participating at the Special Semester on Stochastic with Emphasis on Finance, September 3 - December 5, 2008, organized by RICAM (Johann Radon Institute for Computational and Applied Mathematics, Austrian Academy of Sciences), Linz, Austria.

The second author also thanks the Department of Knowledge-Based Mathematical Systems (Linz, Austria) for the kind hospitality. 


\section{References}

Alvoni, E., Papini, P.-L., and Spizzichino, F. (2009). On a class of transformations of copulas and quasi-copulas. Fuzzy Sets and Systems, 160(3):334-343.

Arnold, B. C., Castillo, E., and Sarabia, J. M. (2008). Multivariate distributions defined in terms of contours. J. Statist. Plan. Infer., 138(12):4158-4171.

Avérous, J. and Dortet-Bernadet, J.-L. (2004). Dependence for Archimedean copulas and aging properties of their generating functions. Sankhya, 66(4):607-620.

Bassan, B. and Spizzichino, F. (2001). Dependence and multivariate aging: the role of level sets of the survival function. In System and Bayesian reliability, volume 5 of Ser. Qual. Reliab. Eng. Stat., pages 229-242. World Sci. Publ., River Edge, NJ.

Bassan, B. and Spizzichino, F. (2005a). Bivariate survival models with Clayton aging functions. Insurance Math. Econom., 37(1):6-12.

Bassan, B. and Spizzichino, F. (2005b). Relations among univariate aging, bivariate aging and dependence for exchangeable lifetimes. J. Multivariate Anal., 93(2):313-339.

Chan, W., Proschan, F., and Sethuraman, J. (1990). Convex-ordering among functions, with applications to reliability and mathematical statistics. In Topics in statistical dependence (Somerset, PA, 1987), volume 16 of IMS Lecture Notes Monogr. Ser., pages 121-134. Inst. Math. Statist., Hayward, CA.

Charpentier, A. (2008). Dynamic dependence ordering for Archimedean copulas and distorted copulas. Kybernetika (Prague), 44(6):777-794.

Charpentier, A. and Segers, J. (2007). Lower tail dependence for Archimedean copulas: characterizations and pitfalls. Insurance Math. Econom., 40(3):525-532.

Charpentier, A. and Segers, J. (2009). Tails of multivariate Archimedean copulas. J. Multivariate Anal., 100(7):1521-1537.

Crane, G. and van der Hoek, J. (2008). Using distortions of copulas to price synthetic CDOs. Insurance Math. Econom., 42(3):903-908.

Denneberg, D. (1994). Non-additive Measure and Integral. Kluwer, Dordrecht.

Durante, F. (2006). A new class of symmetric bivariate copulas. J. Nonparametr. Stat., 18(7-8):499-510.

Durante, F., Kolesárová, A., Mesiar, R., and Sempi, C. (2008). Semilinear copulas. Fuzzy Sets and Systems, 159(1):63-76.

Durante, F. and Sempi, C. (2005a). Copula and semicopula transforms. Int. J. Math. Math. Sci., 2005(4):645-655.

Durante, F. and Sempi, C. (2005b). Semicopulæ. Kybernetika (Prague), 41(3):315-328.

Durante, F. and Spizzichino, F. (2009). Semi-copulas, capacities and families of level curves. Fuzzy Sets and Systems, in press. 
Durrleman, V., Nikeghbali, A., and Roncalli, T. (2000). A simple transformation of copulas. Technical report.

Frees, E. W. and Valdez, E. A. (1998). Understanding relationships using copulas. $N$. Am. Actuar. J., 2(1):1-25.

Genest, C. and Rivest, L.-P. (2001). On the multivariate probability integral transformation. Statist. Probab. Lett., 53(4):391-399.

Hardy, G. H., Littlewood, J. E., and Pólya, G. (1952). Inequalities. Cambridge University Press. 2d ed.

Harris, R. (1970). A multivariate definition for increasing hazard rate distribution functions. Ann. Math. Statist., 41:713-717.

Joe, H. (1997). Multivariate models and dependence concepts, volume 73 of Monographs on Statistics and Applied Probability. Chapman \& Hall, London.

Juri, A. and Wüthrich, M. V. (2002). Copula convergence theorems for tail events. Insurance Math. Econom., 30(3):405-420.

Juri, A. and Wüthrich, M. V. (2003). Tail dependence from a distributional point of view. Extremes, 6(3):213-246.

Karlin, S. and Rinott, Y. (1980a). Classes of orderings of measures and related correlation inequalities. I. Multivariate totally positive distributions. J. Multivariate Anal., 10(4):467-498.

Karlin, S. and Rinott, Y. (1980b). Classes of orderings of measures and related correlation inequalities. II. Multivariate reverse rule distributions. J. Multivariate Anal., 10(4):499516.

Klement, E. P., Mesiar, R., and Pap, E. (2005a). Archimax copulas and invariance under transformations. C. R. Math. Acad. Sci. Paris, 340(10):755-758.

Klement, E. P., Mesiar, R., and Pap, E. (2005b). Transformations of copulas. Kybernetika (Prague), 41(4):425-434.

Lai, C.-D. and Xie, M. (2006). Stochastic ageing and dependence for reliability. Springer, New York.

Marshall, A. W. and Olkin, I. (1979). Inequalities: theory of majorization and its applications, volume 143 of Mathematics in Science and Engineering. Academic Press Inc. [Harcourt Brace Jovanovich Publishers], New York.

McNeil, A. J., Frey, R., and Embrechts, P. (2005). Quantitative risk management. Concepts, techniques and tools. Princeton Series in Finance. Princeton University Press, Princeton, NJ.

Mehmet, Y. and Yalçın, T. (2007). Convex ordering for bivariate distributions. Comm. Statist. Theory Methods, 36(1-4):465-472. 
Morillas, P. M. (2005). A method to obtain new copulas from a given one. Metrika, $61(2): 169-184$.

Müller, A. and Stoyan, D. (2002). Comparison methods for stochastic models and risks. Wiley Series in Probability and Statistics. John Wiley \& Sons Ltd., Chichester.

Nappo, G. and Spizzichino, F. (2009). Kendall distributions and level sets in bivariate exchangeable survival models. Inform. Sci., in press.

Nelsen, R. B. (2006). An introduction to copulas. Springer Series in Statistics. Springer, New York, second edition.

Salvadori, G., De Michele, C., Kottegoda, N. T., and Rosso, R. (2007). Extremes in Nature. An Approach Using Copulas, volume 56 of Water Science and Technology Library. Springer, Dordrecht (NL).

Saminger-Platz, S., De Baets, B., and De Meyer, H. (2008). A generalization of the Mulholland inequality for continuous Archimedean t-norms. J. Math. Anal. Appl., 345(2):607-614.

Schweizer, B. and Sklar, A. (2006). Probabilistic Metric Spaces. Dover Publications, Mineola, N. Y.

Shaked, M. and Shanthikumar, J. G. (2007). Stochastic orders. Springer Series in Statistics. Springer, New York. 